\title{
Rheumatoid factors specific for active rheumatoid arthritis
}

\author{
Dean T Noritake, Keith K Colburn, Grace Chan, Richard H Weisbart
}

\begin{abstract}
To measure rheumatoid factors specific for patients with rheumatoid arthritis an enzyme linked immunosorbent assay (ELISA) was developed to measure rheumatoid factors in human serum that bind a cross-reactive determinant shared on human and other mammalian IgG. Rheumatoid factors that cross link human IgG and sheep IgG in a double binding ELISA were almost completely specific (>99\%) for rheumatoid arthritis in assays of 108 sera from patients with rheumatoid arthritis compared with 231 sera from patients with other connective tissue diseases and 365 sera from healthy subjects and patients without these diseases. Moreover, positive tests occurred primarily in patients with active arthritis $(r=0.68)$. In contrast, these rheumatoid factor autoantibodies were not detected in sera from most of the patients with other autoimmune diseases, including patients with systemic lupus erythematosus. These results show that rheumatoid factors identified in human sera by the double binding test are specific for active rheumatoid arthritis.
\end{abstract}

Rheumatoid factors are antiglobulin antibodies that bind heterogeneous determinants on the crystallisable fragment $(\mathrm{Fc})$ of IgG immunoglobulins, and are found in the serum and synovial fluid of most patients with rheumatoid arthritis. ${ }^{1-3}$ Rheumatoid factors are also present in patients with various other chronic diseases, however. The binding specificity of rheumatoid factors includes allotypic antigens $(\mathrm{Gm})$ on human IgG,${ }^{4}$ neoantigens created within IgG by the formation of immune complexes, ${ }^{5-7}$ and cross-reactive antigens shared by other mammalian IgG immunoglobulins. ${ }^{8}$ Rheumatoid factors that bind alloantigens may occur as a result of blood transfusions and pregnancy, but they do not represent true autoantibodies unless they bind self determinants. ${ }^{9}$ Rheumatoid factors that bind neoantigens are also not true autoantibodies as they are directed to new determinants formed within immune complexes. In contrast, Allen and Kunkel showed the presence of an autologous reactive rheumatoid factor specificity, $\mathrm{Ga}$, in rheumatoid serum. ${ }^{10}$ The presence of autoreactive rheumatoid factors in circulating immune complexes from patients with rheumatoid arthritis supports a potential pathogenic role for these autoantibodies. The methods required to measure rheumatoid factor autoantibodies are too complex to permit an evaluation of their specificity for rheumatoid arthritis, however.
We recently produced a human monoclonal IgG rheumatoid factor (mAb hRF-1) from synovial tissue of a patient with rheumatoid-like arthritis that bound multiple mammalian IgG immunoglobulins. ${ }^{11}$ This finding showed that human IgG may share cross-reactive determinants on other mammalian IgG immunoglobulins. The binding of rheumatoid factors to different mammalian IgG immunoglobulins might be the result of such shared antigens rather than the presence of diverse antibody specificities as previously assumed. ${ }^{12}$ We decided, therefore, to characterise crossreactive determinants on mammalian IgG immunoglobulins in order to identify those which may be specific for rheumatoid arthritis. In preliminary studies we showed that rheumatoid factors to sheep IgG and native human IgG have the best combined sensitivity and specificity for rheumatoid arthritis. Therefore, we developed an enzyme linked immunosorbent assay (ELISA) that measures serum rheumatoid factors that simultaneously bind and cross link human IgG and sheep IgG. We then used this assay to determine the sensitivity and specificity for rheumatoid arthritis of human serum rheumatoid factors that bind a cross-reactive determinant on human and sheep IgG.

\section{Subjects and methods}

SUBJECTS

Serum was obtained from 704 patients, including 108 with classical rheumatoid arthritis defined by revised criteria of the American Rheumatism Association ${ }^{13}$; 231 patients with connective tissue diseases other than rheumatoid arthritis, including 190 with systemic lupus erythematosus, 31 with progressive systemic sclerosis, three with polymyositis, four with temporal arteritis, and three with polyarteritis nodosa; 317 patients in hospital with nonrheumatic diseases; and 48 healthy subjects. Serum samples were stored at $-20^{\circ} \mathrm{C}$ until assayed.

A prospective study was done to evaluate the relation between rheumatoid factors and rheumatoid arthritis disease activity. Rheumatoid arthritis disease activity was evaluated by doctors in $\mathbf{4 0}$ patients at the time serum was obtained. An index of disease activity was based on the number of swollen or tender joints, the patient's subjective evaluation of pain, and a quantitative estimate of the duration of morning stiffness. Each variable was given a value from zero (inactive) to five (most active), and the activity index was recorded as the sum of the three values. A Westergren erythrocyte sedi- 
mentation rate was obtained in 23 of these patients as an additional objective measure of disease activity.

\section{RHEUMATOID FACTORS MEASURED BY THE} AGGLUTINATION OF LATEX PARTICLES

All serum samples were assayed for rheumatoid factors by the agglutination of latex beads coated with heat aggregated human IgG (RF test; Difco, Detroit, MI). Serum samples were assayed in twofold serial dilutions beginning at $1 / 20$, and positive tests were selected as those with a titre of $1 / 160$ or greater. By choosing a titre of $1 / 160$ we eliminated low titre responses that occurred more commonly in patients without rheumatoid arthritis. Moreover, at titres of $1 / 160$ or greater the positive rheumatoid factor tests in patients without rheumatoid arthritis were comparable with those found in patients with rheumatoid arthritis.

\section{RHEUMATOID FACTORS MEASURED BY ELISA}

Serum samples that agglutinated latex particles were also assayed by an ELISA using 96-well microtitre plates coated overnight at $4^{\circ} \mathrm{C}$ with 10 $\mu \mathrm{g} / \mathrm{ml}$ purified native human IgG, human IgG aggregated by heating to $63^{\circ} \mathrm{C}$ for 30 minutes, and purified mammalian IgG immunoglobulins, including sheep, horse, rabbit, mouse, guinea pig, and goat IgG. The plates were washed three times with phosphate buffered saline containing 0.05\% Tween-20 (PBST), and human serum $(0.1 \mathrm{ml})$ diluted $1 / 100$ with $1 \%$ ovalbumin was incubated in the wells overnight at $4^{\circ} \mathrm{C}$. The plates were washed with PBST and affinity purified alkaline phosphatase conjugated goat antibodies specific for human IgM Fc were added for one hour at room temperature. Preliminary tests showed that the conjugated antiserum used did not competitively inhibit the rheumatoid factor assay. After the wells had been washed with PBST, alkaline phosphatase was measured by the absorbance at $405 \mathrm{~nm}$ due to conversion of $p$-nitrophenyl phosphate to $p$ nitrophenol.

\section{RHEUMATOID FACTORS MEASURED BY THE DOUBLE} BINDING TEST

Serum samples from all 704 subjects were assayed for rheumatoid factors with autologous binding or simultaneous binding, or both, to human IgG and sheep IgG. Human IgG was biotinylated using NHS-LC-biotin (Pierce, Rockford, Il). Plates were coated with sheep IgG, and human serum (diluted 1:100 with ovalbumin) together with $0.625 \mu \mathrm{g}$ of biotinylated normal human IgG was added to each well which was incubated overnight at $4^{\circ} \mathrm{C}$. Human antibodies measured by this double binding assay might be those that simultaneously bind immobilised sheep IgG and soluble biotinylated pooled normal human IgG (fig 1A), or human rheumatoid factor autoantibodies that cross link biotinylated human IgG and autologous IgG bound to sheep IgG (fig 1B). The presence of biotinylated human IgG bound to the plate was measured by adding peroxidase conjugated strepavidin, washing the plates with PBST, and

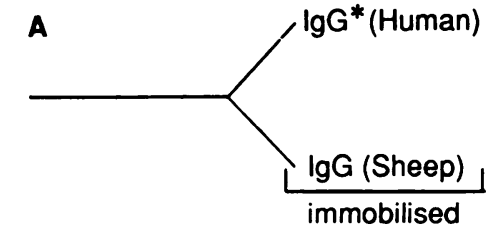

Figure 1 Rheumatoid factors measured by enzyme linked immunosorbent assay (ELISA) in the double binding test. Panel A shows cross linking of immobilised sheep IgG and soluble biotinylated $\left(^{*}\right)$ pooled normal human IgG by a serum rheumatoid factor. Panel $B$ shows cross linking of autologous IgG bound to immobilised sheep IgG and soluble biotinylated normal human IgG by a serum rheumatoid factor.

observing the conversion of $2,2^{\prime}$-azinobis(3ethylbenzthiazoline) sulphonate to a chromophore with an optical density (absorbance) maximum at $414 \mathrm{~nm}$. A negative control was included in each assay by measurement of binding to a plate coated with ovalbumin alone. A known positive serum from a patient with rheumatoid arthritis was used as a positive control in each assay, and the results were expressed as a percentage of the positive control.

\section{STATISTICAL ANALYSIS}

The precision of the double binding test for rheumatoid factor was evaluated by intrabatch and interbatch comparisons and expressed as the coefficient of variation, $V$, where $V=$ standard deviation $(\sigma) /$ mean $(\mu)$.

\section{Results}

RHEUMATOID FACTORS MEASURED BY THE AGGLUTINATION OF LATEX PARTICLES

Serum samples were assayed in twofold serial dilutions beginning at $1 / 20$. To compare sera with comparable levels of rheumatoid factor in patients with and without rheumatoid arthritis a serum dilution of $1 / 160$ was selected for inclusion as a positive test. At a dilution of $1 / 160$ serum from $41 / 108(38 \%)$ patients with rheumatoid arthritis agglutinated IgG coated latex particles. In contrast, 14/231 (6\%) patients with other connective tissue diseases, primarily systemic lupus erythematosus, and 19/317 (6\%) patients with non-rheumatic diseases had positive rheumatoid factor tests (total positive tests $33 / 548(6 \%)$ ). The median titre of positive responses in both those with and without rheumatoid arthritis was $1 / 320$. The latex agglutination test was, therefore, $38 \%$ sensitive and $94 \%$ specific for rheumatoid arthritis when serum samples were assayed at dilutions of $1 / 160$ or greater. 
RHEUMATOID FACTORS MEASURED BY THE DOUBLE BINDING TEST

In preliminary experiments native human IgG and sheep IgG gave the best overall results for both sensitivity and specificity for rheumatoid arthritis, so they were selected for use in the double binding assay.

Rheumatoid factors in serum from 704 subjects were assayed for binding to autologous IgG, or by their ability to bind simultaneously immobilised sheep IgG and soluble biotinylated human IgG in a double binding assay. Positive tests were defined as binding greater than $28 \%$ of a standard positive control as $28 \%$ corresponded to $2 \mathrm{SD}$ above the mean response for the control group of 548 patients without rheumatoid arthritis. The results of the double binding test showed that it was comparable in sensitivity $(39 / 108(36 \%))$ to the latex agglutination test $(41 / 108(38 \%))$. In contrast with the latex agglutination test, the double binding test was considerably more specific as positive tests occurred in only $2 / 317(0.6 \%)$ of patients with non-rheumatic diseases, in only $3 / 231(1 \cdot 3 \%)$ patients with rheumatic diseases other than rheumatoid arthritis, and in 0/48 healthy subjects. The total number of positive tests in subjects without rheumatoid arthritis was $5 / 596$, or only $0.8 \%$ (fig 2 ). The double binding test was, therefore, $99.2 \%$ specific for rheumatoid arthritis compared with only $94 \%$ specificity in the latex agglutination test $\left(\chi^{2}=24 \cdot 2, p<0 \cdot 001\right)$. Based on a prevalence rate of $1.0 \%$ for rheumatoid arthritis, the positive predictive value for the latex agglutination test for serum diluted $1 / 160$ is $6 \%$ compared with $31.3 \%$ for the double binding ELISA using both sheep IgG and human IgG. The double binding test was not positive in any patients without rheumatoid arthritis with latex agglutination titres of less than $1 / 160$.

RHEUMATOID FACTOR TESTS IN PATIENTS WITHOUT RHEUMATIC DISEASES

Serum from 19/317 patients without rheumatic

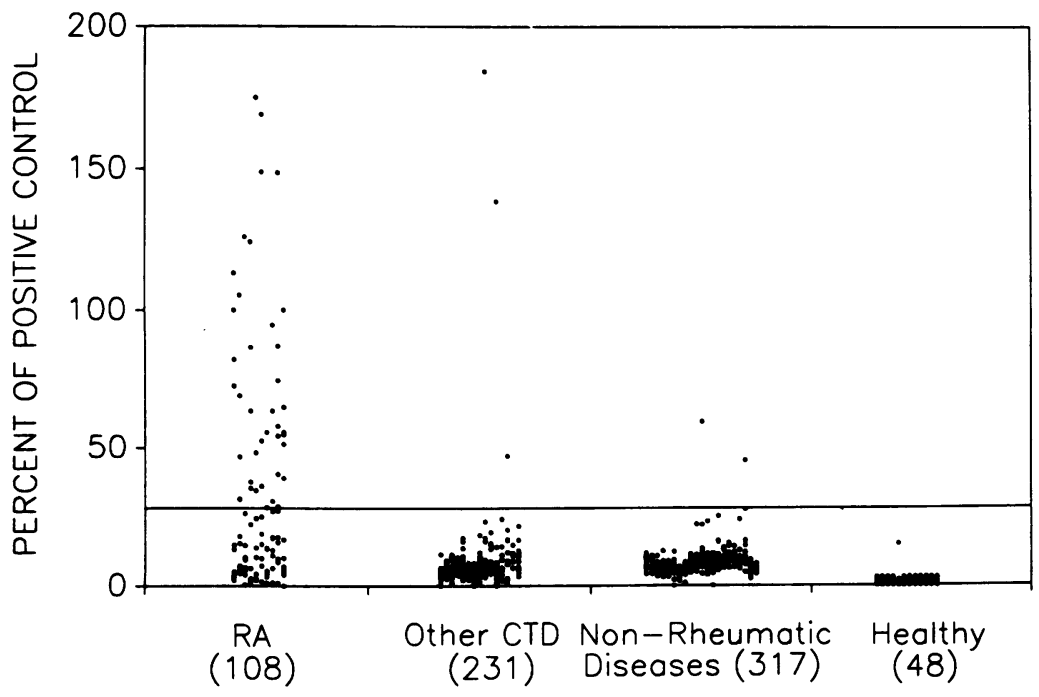

Figure 2 Rheumatoid factors measured in the double binding test. Rheumatoid factors were measured that bind autologous IgG or that simultaneously bind immobilised sheep IgG and moluble biotinylated pooled normal human IgG in an ELISA.RA=rheumatoid arthritis, other $C T D=$ connective tissue diseases other than rheumatoid arthritis. The number of patients in each group is indicated.
Table 1 Clinical diagnoses of patients without rheumatoid arthritis with serum rheumatoid factors measured by latex agglutination

\begin{tabular}{ll}
\hline Clinical diagnosis & $\begin{array}{l}\text { Number positive } \\
(\text { titres }>1 / 160)\end{array}$ \\
\hline $\begin{array}{l}\text { Non-connective tissue diseases } \\
\text { Hepatitis or cirrhosis }\end{array}$ & 7 \\
Diabetes mellitus & 1 \\
Schizophrenia & 1 \\
Peripheral vascular disease & 2 \\
Gout & 2 \\
Inflammatory bowel disease & 1 \\
Ankylosing spondylitis & 1 \\
Psoriasis & 1 \\
Bacterial endocarditis & 1 \\
Malignancy & 1 \\
Intravenous drug abuse & 1 \\
Connective tissue diseases & \\
Systemic lupus erythematosus & 12 \\
Systemic sclerosis & 1 \\
Polymyositis & 1 \\
\hline
\end{tabular}

disease with positive rheumatoid factors by latex agglutination and with titres comparable with those of patients with rheumatoid arthritis were assayed for IgM rheumatoid factors by ELISA. Several of these patients (7/19) had liver disease (table 1). We characterised the binding specificities of these rheumatoid factors by comparing their binding to heat aggregated human IgG, native human IgG, sheep IgG, and simultaneous binding to sheep and human IgG. Serum from 18/19 patients contained IgM antibodies that bound human heat aggregated IgG when assayed by ELISA, results that were comparable with the latex agglutination test using aggregated IgG (fig 3). In contrast, there were fewer positive tests using native human $\operatorname{IgG}\left(9 / 19, \chi^{2}=8 \cdot 2, \mathrm{p}<0.01\right)$, sheep $\operatorname{IgG}(12 / 19$, $\left.\chi^{2}=4 \cdot 8, p=0.028\right)$, and human/sheep IgG $(2 / 19$, $\left.\chi^{2}=23 \cdot 8, \mathrm{p}<0.001\right)$. Moreover, double binding to sheep and human IgG produced fewer positive tests than human IgG or sheep IgG alone $\left(\chi^{2}=4.6, p=0.032\right.$, and $\chi^{2}=8.3, p<0.01$ respectively). The double binding assay was positive in one patient with bacterial endocarditis and one patient with liver disease. There was no correlation between the rheumatoid factor ELISA using aggregated IgG and the double binding test $(r=0.39)$, showing that the difference in the results between these tests was not due simply to a change in assay sensitivity.

RHEUMATOID FACTOR TESTS IN PATIENTS WITH CONNECTIVE TISSUE DISEASES OTHER THAN RA Serum samples from 14/231 patients with connective tissue diseases other than rheumatoid arthritis with positive rheumatoid factors by latex agglutination and with titres comparable with those for patients with rheumatoid arthritis were tested for rheumatoid factors by ELISA. Most of the positive rheumatoid factor tests occurred in patients with systemic lupus erythematosus (12/14) (table 1). In contrast with patients without connective tissue diseases, these patients with rheumatic disease produced IgM antibodies that bound equally well to heat aggregated human IgG, native human IgG, and sheep IgG (fig 4). The ELISA responses to aggregated IgG (18/19 positive) were comparable with the latex agglutination test using aggregated IgG. The rheumatoid factors in 


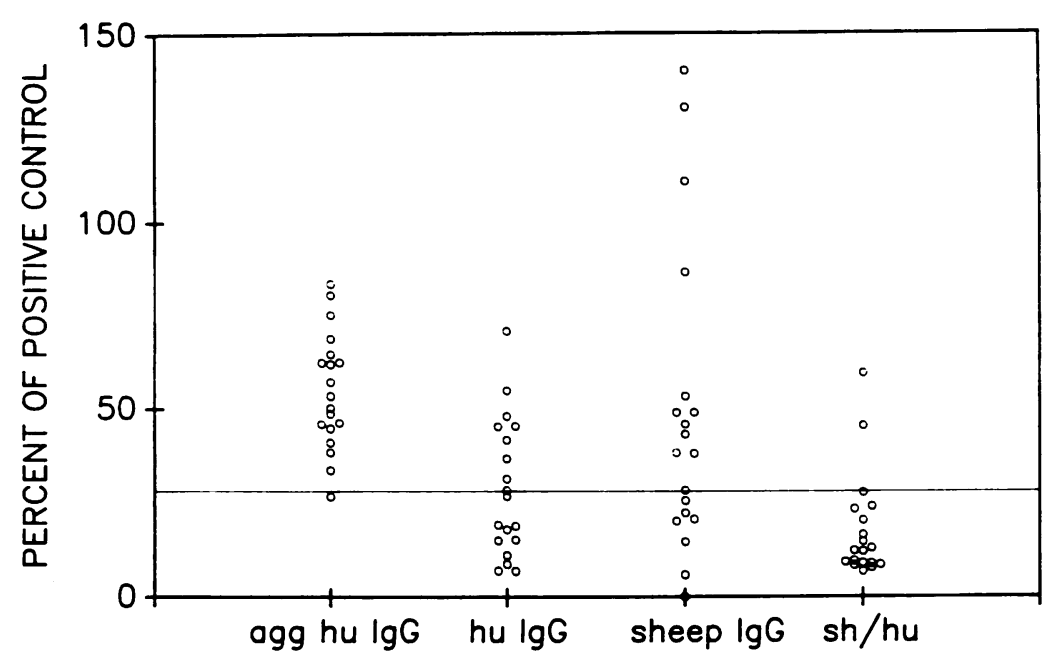

Figure 3 Rheumatoid factors measured by ELISA in patients without rheumatic disease. Agg hu IgG = heat aggregated IgG; hu IgG = native human IgG; sh/hu=simultaneous binding to sheep IgG and human IgG.

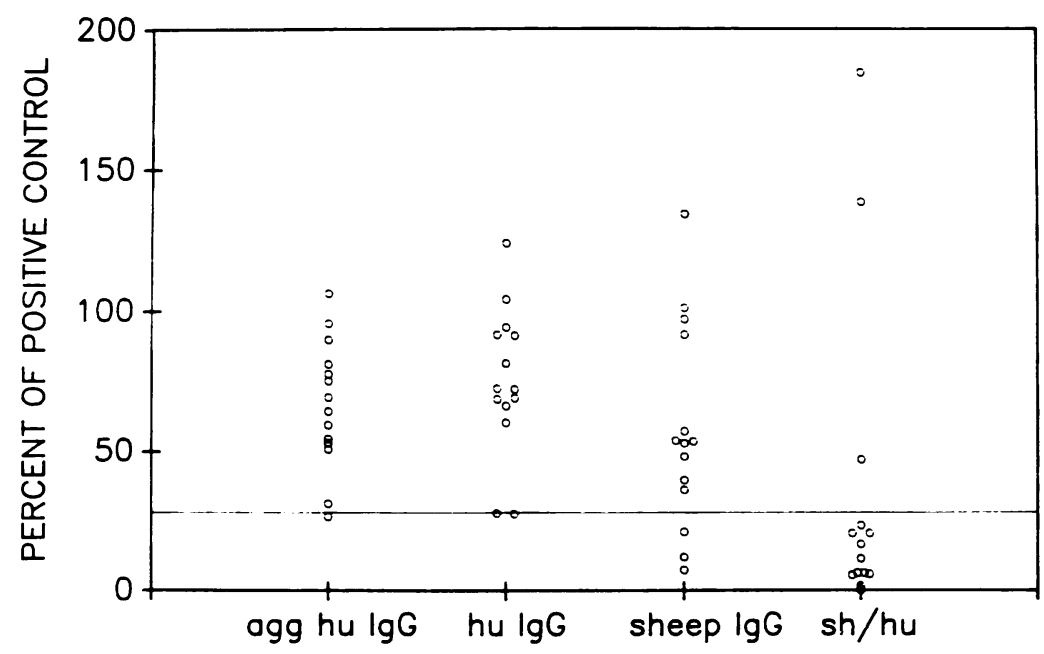

Figure 4 Rheumatoid factors measured by ELISA in patients with connective tissue diseases other than rheumatoid arthritis. The abbreviations are the same as those in fig 3.

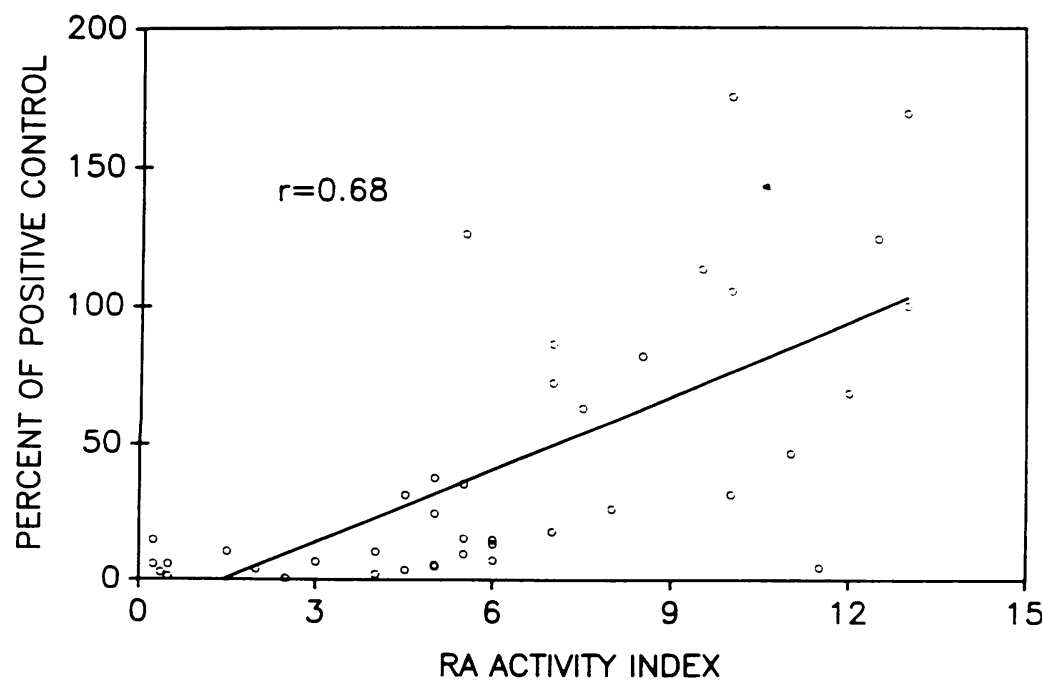

Figure 5 Correlation of rheumatoid arthritis disease activity with the level of serum rheumatoid factors measured by the double binding ELISA test. $r=$ correlation coefficient, $R A=$ rheumatoid arthritis.
Table 2 Clinical characteristics of patients with rheumatoid arthritis evaluated for disease activity

\begin{tabular}{ll}
\hline Number & 40 \\
Female & 16 \\
Male & 24 \\
Mean age (years) & 61 \\
Range & $29-78$ \\
Duration (years) & 16 \\
Range & $1-47$ \\
No with rheumatoid nodules & 12 \\
No receiving DMARDs* & 35 \\
No receiving prednisone & 9
\end{tabular}

*DMARDs= disease modifying antirheumatic drugs. Patients receiving DMARDs included 16 receiving gold, four D-penicillamine, seven methotrexate, and eight azathioprine.

these lupus patients could, however, be distinguished from those in patients with rheumatoid arthritis by their inability to bind and cross link simultaneously sheep IgG and human IgG or their inability to bind autologous IgG that was bound to sheep IgG. For example, the double binding test was positive in fewer lupus patients (3/14) than tests using aggregated IgG $\left(13 / 14, \chi^{2}=11 \cdot 8, p<0.001\right)$, native human IgG $\left(12 / 14, \chi^{2}=9 \cdot 2, p<0.01\right)$, or sheep IgG $(11 / 14$, $\chi^{2}=7 \cdot 0, p<0.01$ ) (fig 4). These results indicate that many patients without rheumatoid arthritis produce antibodies to sheep IgG, but these antibodies do not bind determinants that cross react with determinants shared with human IgG.

\section{CORRELATION OF RHEUMATOID FACTORS WITH RA} DISEASE ACTIVITY

Rheumatoid arthritis disease activity was evaluated in $\mathbf{4 0}$ patients and compared with serum rheumatoid factor levels. Patients representing a broad spectrum of disease activity were chosen. Table 2 shows the clinical characteristics of these patients. The male predominance reflects, in part, the population of the Veterans Administration Medical Center. Serum rheumatoid factor titres were assayed in serial dilutions by the latex agglutination test, and these rheumatoid factor titres did not correlate well with rheumatoid disease activity $(r=0 \cdot 31)$. In contrast, the levels of rheumatoid factors measured by ELISA in the double binding test did correlate well $(r=0.68)$ with rheumatoid disease activity, indicating that positive tests in the double binding assay occurred primarily in patients with rheumatoid arthritis who had active disease (fig 5). For example, 0/11 patients with minimal disease activity (activity index $<4)$ were positive in the double binding test compared with $14 / 16(88 \%)$ patients with moderate to severe disease (activity index $>6$ ). Positive tests were defined as those greater than $28 \%$ of the positive control, corresponding to more than 2 SD above the mean for the 548 patients without rheumatoid arthritis. Rheumatoid factors by the double binding test also correlated well $(r=0 \cdot 7)$ with the Westergren erythrocyte sedimentation rate in 23 of these patients with rheumatoid arthritis in whom the sedimentation rate was determined (fig 6). These results further support the association of positive rheumatoid factors measured by the double binding test with active rheumatoid arthritis. 


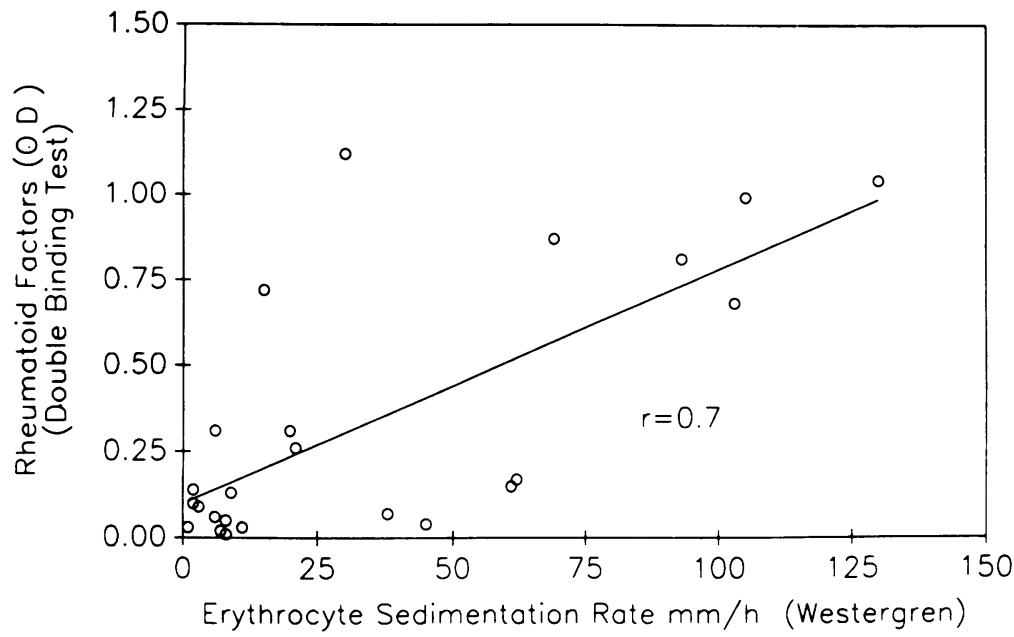

Figure 6 Correlation of rheumatoid factors measured by the double binding test with the Westergren erythrocyte sedimentation rate. $r=$ correlation coefficient.

PRECISION OF THE DOUBLE BINDING TEST FOR RHEUMATOID FACTOR

Replicate tests for rheumatoid factor were done using the double binding test to assess the precision of this ELISA method. Intrabatch variation was measured by testing sera with a low, medium, and high response in six replicates each. The tests were performed as a quadruplicate determination on one 96-well plate, and two sets of duplicate determinations each done on separate 96-well plates. The mean (SD) (measured as optical density) of six replicates for the high, medium, and low response serum was $1.56(0.13), 1.38(0.09)$, and $0.77(0.10)$ respectively; the coefficient of variation for the three groups of serum samples was $0.08,0.06$, and 0.13 respectively. Interbatch variation was measured by testing two positive samples on five separate occasions. The mean (SD) for each group of five determinations was $1 \cdot 38(0 \cdot 14)$ and $1.29(0 \cdot 25)$. The coefficient of variation for each of these interbatch comparisons was $0 \cdot 10$ and $0 \cdot 19$ respectively.

\section{Discussion}

Rheumatoid factors are antiglobulins that bind heterogeneous determinants on IgG Fc and are found in the serum of most patients with rheumatoid arthritis. In contrast with most rheumatoid factors that bind allotypic antigens and neoantigens, rheumatoid factors similar to those that bind the Ga determinant are true autoantibodies, but their specificity for rheumatoid arthritis has not been determined. The presence of these rheumatoid factors in circulating immune complexes of patients with rheumatoid arthritis suggests that they might participate in the pathogenesis of this disease. Methods to measure rheumatoid factor autoantibodies include the use of autologous native IgG or the detection of rheumatoid factors in immune complexes, but these methods are too cumbersome and have not been used to study large numbers of patients. We designed a method that obviated these difficulties and permitted us to measure rheumatoid factor autoantibodies by a simple enzyme linked immunoassay.
We recently produced a human monoclonal IgG rheumatoid factor (mAb hRF-1) that bound multiple mammalian IgG immunoglobulins, indicating that autoimmune rheumatoid factors may bind cross-reactive determinants present on different mammalian IgG. Although previous studies suggest that rheumatoid factors that bind rabbit IgG immunoglobulins in latex agglutination and haemagglutination assays have increased specificity for rheumatoid arthritis, ${ }^{5} 121415$ our studies using an enzyme linked assay did not confirm these results (data not shown). Our studies with multiple mammalian IgG immunoglobulins showed that native human IgG and sheep IgG gave the best overall sensitivity and specificity for rheumatoid arthritis. When we studied patients with other connective tissue diseases, such as system lupus erythematosus, however, rheumatoid factors to native human IgG and sheep IgG could not be distinguished from those in patients with rheumatoid arthritis. Therefore, we designed a simple ELISA for rheumatoid factors that simultaneously bind and cross link immobilised sheep IgG and soluble human IgG. In the presence of $\operatorname{IgG}$ antibodies to sheep IgG this assay measures rheumatoid factors that bind autologous IgG as well as pooled normal human IgG.

Our results show that the double binding ELISA using sheep and human IgG is considerably more specific for rheumatoid arthritis than the latex agglutination test when evaluated at a comparable level of sensitivity. Moreover, we show that most of the rheumatoid factors in patients without rheumatic diseases are not detected by the double binding test. Most of the positive rheumatoid factor tests in patients with connective tissue diseases other than rheumatoid arthritis were found in patients with systemic lupus erythematosus. Although patients with systemic lupus erythematosus produced antiglobulin antibodies that bound native human IgG and sheep IgG, most did not produce antibodies that could simultaneously bind both human IgG and sheep IgG, indicating that they did not bind a cross-reactive determinant. Furthermore, these patients did not seem to produce antibodies to autologous IgG as the double binding assay was negative even in the presence of IgG antibodies to sheep IgG. Most patients with lupus who are positive for rheumatoid factor by latex agglutination, therefore, do not seem to produce true autoantibodies.

The double binding assay measures a variety of autoantibody specificities, including those which are related to $\mathrm{Ga}$ and $\mathrm{Gm}$. Autoantibodies of different specificity might be responsible for the presence of occasional 'false positive' tests in patients without rheumatoid arthritis.

Nevertheless, our findings establish the relative specificity of rheumatoid factors that are true autoantibodies, such as Ga related rheumatoid factors, for rheumatoid arthritis. Rheumatoid factors that were true autoantibodies were specific $(>99 \%)$ for rheumatoid arthritis. Although these autoantibodies were only $36 \%$ sensitive for rheumatoid arthritis in 108 patients, they were $88 \%$ sensitive for patients with moderate to severe rheumatoid arthritis in the 
40 patients whose disease activity was determined.

The double binding test reduces the incidence of false positive tests fivefold compared with latex agglutination test, and may, therefore, be useful as a diagnostic test for rheumatoid arthritis. In addition, our studies identify a cross-reactive determinant on human IgG and sheep IgG that may facilitate studies to identify and molecularly characterise disease related rheumatoid factors. Further studies of rheumatoid factor autoantibodies specific for rheumatoid arthritis may help to establish their role in the pathogenesis of rheumatoid arthritis.

We thank Amelia Kacena for expert technical help, Dr Arnold Bayer, and Dr Robert Morris for providing serum from their patients, and Romaine Saxton for helpful discussions and editorial assistance.

1 Waaler E. On the occurrence of a factor in human serum activating the specific agglutination of sheep blood corpuscles. Acta Pathologica, Microbiologica, et Scandinavica 1940; 17: 172-88.

2 Rose H M, Ragan C, Pearce E, Lipman M O. Differential agglutination of normal and sensitized sheep erythrocytes by sera of patients with rheumatoid arthritis. Proc Soc Exp Biol Med 1948; 68: 1-6.

3 Natvig J B, Gaarder P I, Turner M W. IgG antigens of the C gamma-2 and $C$-gamma-3 homology regions interacting with rheumatoid factors. Clin Exp Immunol 1972; 12: 177-83.

4 Gaarder P I, Natvig J B. The reaction of rheumatoid anti-Gm antibodies with native and aggregated $\mathrm{Gm}$-negative IgG. Scand f Immunol 1974; 3: 559-71.

5 Henney C S, Stanworth D R, Gell P G H. Exposure of new antigenic determinants following antigen-antibody combination. Nature 1965; 205: 1079-81.

6 Johnson P M, Watkins J, Holborow E J. Antiglobulin production to altered IgG in rheumatoid arthritis. Lancet production to

7 Brown E J, Bekisz J. Neoantigens appear in human IgG upon antigen binding: detection by antibodies that react specifically with antigen-bound IgG. F Immunol 1984; 132 $1346-52$

8 Butler V P, Vaughan J H. Hemagglutination by rheumatoid factor of cells coated with animal gamma globulin. Proc Soc Exp Biol Med 1964; 116: 585-93.

9 Steinberg A G, Wilson J A. Hereditary globulin factors and immune tolerance in man. Science 1963; 140: 303-4.

10 Allen J, Kunkel H G. Hidden rheumatoid factors with specificity for native gamma globulins. Arthritis Rheum 1966; 9: 758-68.

11 Weisbart $R$ H, Noritake D T, Colburn $K K$, Saxton $R$ E. Human monoclonal IgG rheumatoid factor has structural Human monoclonal IgG rheumatoid factor has structural homology with bact

12 Carson D A. Rheumatoid factor. In: Kelley W N, Harris E D Jr, Ruddy S, Sledge C B, eds. Textbook of rheumatology. Philadelphia: Saunders, 1981: 685

13 Arnett F C, Edworthy S M, Bloch D A, et al. The American Rheumatism Association 1987 revised criteria for the classification of rheumatoid arthritis. Arthritis Rheum 1988; 31: 315-24.

14 Butler V P, Vaughan J H. The reaction of rheumatoid factor with animal gamma-globulins: quantitative considerations. Immunology 1965; 8: 144-59.

15 Pope R M, McDuffy S J. IgG rheumatoid factor: analysis of various species of IgG for detection by radioimmunoassay. various species of IgG for detection
f Lab Clin Med 1981; $97: 842-53$. 